\title{
GW100: a plane wave perspective for small molecules
}

\author{
Emanuele Maggio, ${ }^{1}$ Peitao Liu, ${ }^{1,2}$ Michiel J. van Setten, ${ }^{3}$ and Georg Kresse ${ }^{1, * *}$ \\ ${ }^{1}$ University of Vienna, Faculty of Physics and Center for Computational \\ Materials Science, Sensengasse 8/12, A-1090 Vienna, Austria \\ ${ }^{2}$ Institute of Metal Research, Chinese Academy of Sciences, Shenyang 110016, China \\ ${ }^{3}$ Nanoscopic Physics, Institute of Condensed Matter and Nanosciences, \\ Université Catholique de Louvain, 1348 Louvain-la-Neuve, Belgium
}

(Dated: November 28, 2016)

\begin{abstract}
In a recent work, van Setten and coworkers have presented a carefully converged $G_{0} W_{0}$ study of 100 closed shell molecules [J. Chem. Theory Comput. 11, 5665 (2015)]. For two different codes they found excellent agreement to within few $10 \mathrm{meV}$ if identical Gaussian basis sets were used. We inspect the same set of molecules using the projector augmented wave method and the Vienna ab initio simulation package (VASP). For the ionization potential, the basis set extrapolated plane wave results agree very well with the Gaussian basis sets, often reaching better than $50 \mathrm{meV}$ agreement. In order to achieve this agreement, we correct for finite basis set errors as well as errors introduced by periodically repeated images. For electron affinities below the vacuum level differences between Gaussian basis sets and VASP are slightly larger. We attribute this to larger basis set extrapolation errors for the Gaussian basis sets. For quasi particle (QP) resonances above the vacuum level, differences between VASP and Gaussian basis sets are, however, found to be substantial. This is tentatively explained by insufficient basis set convergence of the Gaussian type orbital calculations as exemplified for selected test cases.
\end{abstract}

PACS numbers: 71.15.-m, 71.15.Nc., 71.15.Dx, 71.55 Gs

\section{INTRODUCTION}

The $G W$ approximation suggested by Lars Hedin 1 has a long history in solid state physics. First practical applications were already published in the 1980 s by Hanke and coworkers soon followed by the often quoted study of Hybertsen and Louie 2 5. For solids, it is generally found that even the simplest approximation $G_{0} W_{0}$ yields reasonably accurate quasiparticle (QP) energies and band gaps in good agreement with experiment [6] 8 . The results often improve if the Green's function is iterated to self-consistency, either updating the QP-energies only or even the one-electron orbitals 6, 7, 9, 15. Applications of the $G W$ approximation to molecules, however, have been comparatively rare, since codes based on local orbitals, which are by construction particularly well suited to treat molecules, did not incorporate the $G W$ approximation until recently. This has changed, with many local basis set codes, such as FHI-aims, MOLGW, Turbomole, and CP2K now supporting $G W$ calculations 16 22. Also, efficient plane wave codes using a Sternheimer approach, such as ABINIT and West [23, 24], are becoming available. As for solids, carefully converged QP calculations are, however, still comparatively scarce [25].

To fill this gap, Bruneval recently performed systematic studies for about 30 molecules [26]. van Setten and coworkers went one step further and evaluated basis set extrapolated $G W$ QP energies for 100 closed shell molecules using several codes [27]. They found that the $G W$ QP energies of the highest occupied orbital (HOMO)

\footnotetext{
* georg.kresse@univie.ac.at
}

and lowest unoccupied orbital (LUMO) of two local basis set codes, FHI-aims and Turbomole, virtually agree, if identical basis sets are used. In many respects this is not astonishing, since two codes ought to yield the same results, if the computational parameters are identical. The two codes are, however, technically quite different. For instance, they introduce auxiliary basis sets to avoid storing the two-electron four orbital integrals. Furthermore, FHI-aims uses a numerical representation of the Gaussians and calculates the self-energy along the imaginary axis (Wick rotation) requiring an analytic continuation to the real axis. All these factors can introduce small uncertainties. Clearly, the study impressively demonstrates that all these intricacies are well under control, and technically well converged results can be obtained using both codes.

The paper by van Setten et al. 27] also reports results using the $G W$ Berkeley plane wave code [28]. Although agreement of that code with experiment is very good if the plasmon-pole model is used, comparison of the fully frequency dependent $G_{0} W_{0}$ HOMO and LUMO with Gaussian basis set results is less satisfactory. For the considered molecules, the mean absolute difference between Gaussian type orbitals (GTO) and plane waves (PWs) is about $200 \mathrm{meV}$ for the HOMO. We note on passing that the agreement between GTO and other plane wave studies is seemingly superior [23, 24], although, this could be related to the fact that these studies only considered a subset of the $G W 100$ set. The disagreement between the Berkeley $G W$ PW code and GTO codes is certainly slightly disconcerting, since it puts decades of studies using PW based $G W$ calculations into question. Remarkably, on the level of DFT, the reported one-electron en- 
ergies of the HOMO agree to within few $10 \mathrm{meV}$. So how can one understand the much larger discrepancies for $G W$ QP energies?

A partial answer is given by the observation that QP energies converge very slowly with respect to the basis set size, as well established for Gaussian type orbitals [20, 26, 27]. van Setten et al. obtained basis set converged $\mathrm{QP}$ energies by extrapolating against the basis set size or against $1 / C_{n}^{3}$, where $C_{n}$ is the basis set cardinal number 27. Extrapolation was based on def2-SVP, def2-TZVP and def2-QZVP, but even though def2-QZVP constitutes a fairly complete set, the extrapolated values can differ by more than $300 \mathrm{meV}$ from the values at the largest considered basis set. Astonishingly, the reported PW results were not extrapolated to the basis set limit, although a recent work of Klimes et al. shows that the $G W$ QP energies converge like one over the number of plane waves [25] and this behaviour is also confirmed by purely analytical arguments 29. Early evidence of this slow convergence using PWs exist aplenty 30 32. In view of this slow convergence, a brute force approach to predict QP energies seems elusive considering that most codes scale cubically with respect to the number of basis functions. The present work tries to rectify this issue by reporting QP energies using the plane wave code VASP, carefully correcting for basis set incompleteness errors, as detailed in section [1]

Another point that we briefly mention in section III is that the calculation of the poles of the $G_{0} W_{0}$ Green's function can be unphysical, if the initial Green's function yields too small excitation energies. In this case, first linearizing the $G_{0} W_{0}$ self-energy and then determining the poles of the Green's function yields more robust QP energies. We, finally, finish with discussions and our conclusions.

\section{THEORY AND COMPUTATIONAL METHOD}

\section{A. Theory}

$G W$ is a well established perturbative approach to calculate QP energies [1. In the $G W$ approximation, one initiates the calculations using a groundstate DFT calculation to obtain the DFT one-electron orbitals $\phi_{n}$ and the corresponding one-electron energies $\epsilon_{n}$.

The first step in a $G W$ calculation is to determine the DFT Green's functions,

$$
G_{0}\left(\mathbf{r}^{\prime}, \mathbf{r}, \omega\right)=\sum_{n} \frac{\phi_{n}\left(\mathbf{r}^{\prime}\right) \phi_{n}^{*}(\mathbf{r})}{\omega-\epsilon_{n}-i \eta \operatorname{sign}\left(\mu-\epsilon_{n}\right)},
$$

where $\mu$ is the chemical potential of the electrons, and $\eta$ a positive infinitesimal. From the Green's function the independent particle polarizability

$$
\chi\left(\mathbf{r}, \mathbf{r}^{\prime}, t\right)=-\mathrm{i} G_{0}\left(\mathbf{r}, \mathbf{r}^{\prime}, t\right) G_{0}\left(\mathbf{r}^{\prime}, \mathbf{r},-t\right),
$$

and the corresponding screened interaction

$$
W\left(\mathbf{r}, \mathbf{r}^{\prime}, \omega\right)=v\left(\mathbf{r}, \mathbf{r}^{\prime}\right)+v(\mathbf{r}, \mathbf{s}) \chi\left(\mathbf{s}, \mathbf{s}^{\prime}, \omega\right) W\left(\mathbf{s}^{\prime}, \mathbf{r}^{\prime}, \omega\right)
$$

can be determined. Here $v$ is the Coulomb kernel, and integration over repeated spatial coordinates (s and $\left.\mathbf{s}^{\prime}\right)$ is assumed. Furthermore, the Green's functions and polarizabilities in frequency and time domain are related by a Fourier transformation. The final step is to calculate the interacting Green's function

$$
G\left(\mathbf{r}, \mathbf{r}^{\prime}, \omega\right)=\frac{1}{\omega-T-V^{\mathrm{H}}(\mathbf{r}) \delta\left(\mathbf{r}-\mathbf{r}^{\prime}\right)-\Sigma\left(\mathbf{r}, \mathbf{r}^{\prime}, \omega\right)},
$$

where $T$ is the kinetic energy operator, $V^{\mathrm{H}}$ is the Hartreepotential, and $\Sigma\left(\mathbf{r}, \mathbf{r}^{\prime}, t\right)$ is the self-energy in the $G W$ approximation:

$$
\Sigma\left(\mathbf{r}, \mathbf{r}^{\prime}, t\right)=\mathrm{i} G_{0}\left(\mathbf{r}, \mathbf{r}^{\prime}, t\right) W\left(\mathbf{r}, \mathbf{r}^{\prime}, t\right) .
$$

The poles of the Green's function then determine the $\mathrm{QP}$ energies. In principle, this cycle can be continued by evaluating $\chi$ in step (2) using the updated Green's function and iterated to self-consistency. It is also possible to obtain partial self-consistency, for instance, by calculating $W$ once and forever using the DFT orbitals and one electron energies and iterating only the Green's function until it is self-consistent [i.e. iterating only Eqs. (4) and (5)].

The most common approximation is, however, the $G_{0} W_{0}$ approximation e.g. used by Hybertsen and Louie [5]. Instead of the poles of the Green's function, this approximation calculates the nodes of the denominator in Eq. (4)

$$
E_{n}^{\mathrm{QP}}=\operatorname{Re}\left[\left\langle\phi_{n}\left|T+V^{H}(\mathbf{r})+\Sigma\left(E_{n}^{\mathrm{QP}}\right)\right| \phi_{n}\right\rangle\right]
$$

in the basis of the DFT orbitals. Since this involves only the diagonal elements of the self-energy, solutions of this equation are cheaper to determine than poles of the fully interacting Green's function. Obviously this is a good approximation, if the self-energy is diagonally dominant in the basis of the DFT orbitals. As already pointed out by Hybertsen and Louie this is generally the case, although there is some evidence that iterating the DFT orbitals is important [6, 9, 33. This is particularly so for atoms or molecules, since the KS potential and, as a result, the KS orbitals do not decay properly at large distances from the molecule.

The solutions obtained by solving Eq. (6) are labeled as $G_{0} W_{0}$ in the present work. Furthermore, a commonly used approximation is to linearize the the energy dependence in the self-energy in Eq. (6) at the DFT oneelectron energy and determine the nodes of the linearized equation. This yields the following approximate position for the nodes [5, 34]:

$E_{n}^{\mathrm{QP}}-\epsilon_{n}^{\mathrm{DFT}}=Z_{n} \operatorname{Re}\left[\left\langle\phi_{n}\left|T+V^{\mathrm{H}}(\mathbf{r})+\Sigma\left(\epsilon_{n}^{\mathrm{DFT}}\right)-\epsilon_{n}^{\mathrm{DFT}}\right| \phi_{n}\right\rangle\right]$ 
were $Z_{n}$ is related to the derivative of the self-energy at $\epsilon_{n}^{\mathrm{DFT}}$

$$
Z_{n}=\left(1-\left.\frac{\partial \operatorname{Re}\left[\left\langle\phi_{n}|\Sigma(\omega)| \phi_{n}\right\rangle\right]}{\partial \omega}\right|_{\epsilon_{n}^{\mathrm{DFT}}}\right)^{-1} .
$$

The correlation factor $Z_{n}$ can be also related to the amplitude of the corresponding QP peak and is a measure of the degree of correlation. For the HOMO and LUMO of molecules, $Z$ is commonly between $0.7-0.9$, corresponding to a low to very low degree of correlation. Solutions of the linearized equations will be labeled as lin- $G_{0} W_{0}$ in the present work, and the first derivative is evaluated using central difference with the step size of $\Delta= \pm 0.1 \mathrm{eV}$.

\section{B. Technical details}

As in the $G W 100$ paper of van Setten et al. 27, we use the PBE functional for the DFT starting point. However, all calculations include scalar relativistic effects, in contrast to the calculations of van Setten et al. that are based on non-relativistic potentials. The potentials used in the present work are the $G W$ potentials distributed with the latest release of VASP (vasp.5.4), and we followed the recommendations in the VASP manual on which version to use. Generally this means that lower lying semi-core states were not correlated in the calculations, except for the alkali and alkali-earth metals, as well as $\mathrm{Ti}$ and $\mathrm{Ga}$. For He, we found issues with the originally distributed potential. The He_GW potential failed to converge in DFT calculations when the plane wave cutoff was increased, because a ghost state was introduced as the basis set size increased. The potential was slightly modified to remove this problem and will be distributed with the next release. Furthermore, for boron to fluorine the potentials B_GW_new, ..., F_GW_new were used (also already distributed with vasp.5.4). These potentials include $d$ partial waves, whereas the standard $G W$ potentials choose the $d$ potential as the local potential.

The potentials used in this work are not the most accurate $G W$ potentials yet available for VASP. Specifically, we have recently shown that norm-conserving (NC) $G W$ potentials are necessary to predict very accurate QP energies for $3 d, 4 d$ and $5 d$ elements 25 , with the NC potentials generally increasing the QP binding energies. In our experience, such highly accurate potentials are, however, not required in the present case for the following reasons. For $s$ and $p$ elements the standard potentials conserve the norm very well to within about $70 \%$, often even $90 \%$. Furthermore, errors introduced by violating the norm-conservation can only occur at very high scattering energies, since the standard $G W$-PAW potentials predict the scattering properties correctly up to about $400 \mathrm{eV}$. Beyond that energy, the PAW projectors become incomplete. For the elements considered here, we expect that the combination of these two effects means that the results for the HOMO and LUMO will be accurate even though we do not use NC potentials. The only exceptions are copper, neon, fluorine, oxygen and possibly nitrogen. These elements possess strongly localized $3 d$ and $2 p$ orbitals. We will return to this point later.

In the calculations presented here we calculate the Green's function, the screened interaction $W$ as well as the self-energy in imaginary time and frequency. This has several advantages compared to the full real frequency implementation also available in VASP. The fully frequency dependent version along the real axis requires at least 100, but for molecules with their sharp resonances often even several hundred frequency points to converge. Since the boxes considered in this work are quite large, we also need several thousands of plane waves to describe the frequency dependent screened interaction and Green's function accurately. This becomes very quickly prohibitive. In the imaginary frequency, on the other hand, only relatively few frequency points are required. In the calculations presented here, 16 frequency points and the time and frequency grids discussed by Kaltak et $a l$. are used 35, 36. These 16 points were found to be sufficient to converge the QP energies of the HOMO and LUMO to about $10 \mathrm{meV}$ [37. The downside of working in the imaginary frequency domain is that the results along the imaginary frequency axis need to be continued to the real axis. This was done using a (16 point) Padé fit following Thiele's reciprocal difference method based on continued fractions [38. We note that the reported FHI-aims results in Ref. 27 were - with few problematic exceptions - also obtained using 16 parameter Padé fits. These exceptions are $\mathrm{BN}, \mathrm{O}_{3}, \mathrm{BeO}, \mathrm{MgO}$ and $\mathrm{CuCN}$ where many more points were required. For the other molecules, the 16 parameter Padé fits yielded excellent agreement with Turbomole, which calculates the exact $G W$ self-energy along the real axis. Details of our implementation are reported elsewhere 37.

The other crucial issues are basis set extrapolation and convergence with respect to the box size. To obtain basis set converged results, we used a relatively small box, but one that still faithfully reproduces the character of the HOMO and LUMO. For this box, we performed calculations for the default cutoff as specified by the VASP potentials, and calculations for three additional plane wave cutoffs, with the largest calculation corresponding to twice the number of plane waves used in the default setup. These four data points are fitted assuming that the $\mathrm{QP}$ energies as a function of the number of plane waves $N_{\text {pw }}$ converge like

$$
E^{\mathrm{QP}}\left(N_{\mathrm{pw}}\right)=E^{\mathrm{QP}}(\infty)+\frac{C}{N_{\mathrm{pw}}},
$$

where $N_{\mathrm{pw}}$ is the number of plane waves in the basis set 25, 39 41. A four point fit and a two point fit with the largest and smallest PW basis set yielded a maximum difference of $10 \mathrm{meV}$ in the QP energies. To illustrate that the basis set dependence is indeed following a $1 / N_{\mathrm{pw}}$ behavior to great accuracy, we will show data for selected molecules in Sec. IIIB The only subtlety impeding an 
accurate and automatic extrapolation is the use of the Padé fit. The slope of the self-energy can vary somewhat between different calculations causing some variations in the predicted QP energies. Extrapolation from these "noisy" data is difficult and error prone. To circumvent this issue, we perform the extrapolation for the self-energy evaluated at the DFT one-electron energies, specifically on $\Delta E=\operatorname{Re}\left[\left\langle\phi\left|T+V^{\mathrm{H}}+\Sigma\left(\epsilon^{\mathrm{DFT}}\right)\right| \phi\right\rangle\right]-$ $\epsilon^{\mathrm{DFT}}$ instead of $\Delta E=E^{\mathrm{QP}}-\epsilon^{\mathrm{DFT}}$, and scale the correction by the $Z$-factor at the smallest, i.e. default, $\mathrm{PW}$ cutoff. In this way, we neglect variations of the $Z$-factor between different basis sets, but these variations are small and dominated by noise.

A few final comments are in place here. In the calculations presented herein, we calculate all orbitals spanned by the PW basis set. This implies that the number of orbitals also increases as the number of plane waves increases. Second, the kinetic energy cutoff for the response function (ENCUTGW in VASP) is set to $2 / 3$ of the cutoff used for the plane wave basis of the orbitals (ENCUT in VASP). Whenever the PW cutoff for the orbitals is increased, the PW cutoff for the basis set of the response function is increased accordingly. This means that a single parameter, the PW cutoff for the orbitals (ENCUT), entirely controls the accuracy of the calculations (at least with respect to the basis sets). Since all the intermediate control parameters are set automatically by VASP, and since the QP energy corrections converge like one over the number of plane waves and orbitals [25, extrapolation to the infinite basis set limit is straightforward and robust.

Let us now comment on the second point, convergence with respect to the cell size. In plane wave codes, it is common practice to truncate the Coulomb kernel at a certain distance $r_{c}$, say half the box size, so that the periodically repeated orbitals can not screen the central atom. The downside of this approach is that it modifies the Coulomb kernel to become 42

$$
\frac{4 \pi e^{2}}{|\mathbf{g}|^{2}}\left(1-\cos \left(|\mathbf{g}| r_{c}\right)\right),
$$

where $\mathbf{g}$ is a plane wave vector. Obviously, this modifies the Coulomb kernel at large reciprocal lattice vectors $\mathbf{g}$. In test calculations we found that this spoils the previously mentioned basis set extrapolation (8): as one increases the plane wave cutoff, one moves through maxima and minima of the truncated Coulomb kernel, causing superimposed oscillations in the QP energies. Basis set extrapolation becomes then uncontrolled. To deal with the repeated images, we instead resort to the standard trick used in periodic codes: $k \cdot p$ perturbation theory 43 . We calculate the first order change of the orbitals with respect to $k$ 44, and accordingly the head and wings of the polarizability and a correction to the $\mathrm{g} \rightarrow 0$ component of the self-energy. This term corrects the leading monopole-monopole interaction between repeated images, but leaves the monopole-dipole and dipole-dipole interactions uncorrected. These two terms fall off like
$1 / V$ and $1 / V^{2}$, where $V$ is the cell size volume [45]. To deal with this, we perform four calculations at different volumes, with the box size progressively increased by $1 \AA$ and fit the data to

$$
a_{0}+a_{1} / V+a_{2} / V^{2}
$$

For most molecules the corrections are small and only of the order of 10-20 meV, whereas for the alkali dimers and some polar molecules the corrections can be as large as $100-200 \mathrm{meV}$. In these cases the correction is very well described by the theoretical equation. We hope to find a better solution in future work, for instance, an explicit subtraction of monopole and dipole interactions between periodic images. In terms of compute time, however, the additional calculations for smaller boxes only require a modest amount of time: since the total compute time scales quadratic to cubic with respect to the number of plane waves, the calculations scale also quadratic to cubic in the volume. Typically we need $12 \AA$ large boxes to obtain results converged to $20 \mathrm{meV}$ with respect to the box size. The additional smaller volumes used for the extrapolation require only half of the compute time of the largest final box.

The final QP energies reported in the next section were obtained by calculating the PBE one-electron HOMO and LUMO for a $25 \AA$ box at an energy cutoff that is $30 \%$ increased compared to the VASP default values. The vacuum level, evaluated as the Hartree plus ionic potential, was evaluated at the position furthest from the center of the molecule and subtracted from the PBE oneelectron energies. We checked that the DFT one-electron energies are converged to a few meV with this setup. To the DFT one-electron energies, the shift of the QP energies $E^{\mathrm{QP}}-\epsilon^{\mathrm{DFT}}$ for the largest considered box, box size corrections, and basis set corrections as described above are added. It goes without saying that this procedure is rather involved and since errors are expected to accumulate, we estimate that the present predictions are only accurate to about $\pm 50 \mathrm{meV}$, where convergence with cell size is the main source of errors and difficult to estimate precisely.

To give a feeling for the required compute time and computational effort, we need to stress that our plane wave code is mainly designed for solids. Nevertheless, a calculations for $\mathrm{C}_{6} \mathrm{H}_{6}$ in a $10 \AA$ box at the default cutoff takes about 4 hours on a single node with 16 Xeon v2 cores. The compute time stays roughly constant if the box size is increased by $1 \AA$ and the number of cores is simultaneously doubled. Furthermore, the compute time is mostly independent of the number of atoms in the box, but increases cubically with the box size as the total number of plane waves increases linearly with the box size. By comparison Turbomole, using the def2-TZVP basis and the resolution of the identity method, takes 30 minutes on a 12 core AMD opteron 6174 for the response and $G W$ part of the calculation (the time spent for the DFT part is negligible in comparison). 


\section{RESULTS}

\section{A. HOMO for $G W 100$}

Let us first note on the agreement at the level of DFT (not shown). In general, our values agree exceedingly well with the PBE values reported in the supplementary material of Ref. 27. In most cases, our PBE HOMO is located between the basis set extrapolated values and the values obtained with the best basis sets used in the GTO calculations (def2-QZVP). On average, our PW HOMOs agree better with the non basis set extrapolated values with a mean deviation (MD) of $7 \mathrm{meV}$ and a mean absolute deviation (MAD) of $19 \mathrm{meV}$. Compared to the GTO basis set extrapolated values, the MD and MAD are $-25 \mathrm{meV}$ and $30 \mathrm{meV}$ (in both cases, $\mathrm{CH}_{2} \mathrm{CHBr}$ was excluded, see below).

van Setten et al. 27] extrapolated the DFT eigenvalues using a cubic polynomial in the inverse of the basis set cardinal number $\left(C_{n}^{-3}\right)$; we believe that this is not appropriate and will overestimate the basis set corrections. It is commonly agreed that DFT calculations converge exponentially with the cardinal number, whereas any correlated wave function calculation converges with the inverse of the basis set size (corresponding roughly to $C_{n}^{-3}$ ) [25 27]. This is a result of Kato's cusp condition 121 causing a kink in the many-body wave function as two coordinates approach each other. We have shown that this problem carries over to $G W$ calculations [25]. As a one-electron theory, density functional theory does not suffer from this slow convergence. We hence believe that van Setten overestimated the basis set corrections for DFT. This is supported by the observation that our $\mathrm{PBE}$ results tend to be closer to the non-extrapolated Gaussian results at the level of def2-QZVP.

We now turn to the $\mathrm{QP}$ energies predicted at the level of $G_{0} W_{0}$ shown in Table I. The agreement between the VASP PW and the GTO results is generally very good. We note that the $G_{0} W_{0}$ approximation used here is identical to the one applied by van Setten et al. [27]. Specifically, van Setten determined the nodes of Eq. (6), and we do exactly the same in the present work. Linearization of the QP equation (7) yields generally somewhat larger QP energies and often improves agreement with experiment slightly (column lin- $G_{0} W_{0}$ ). This trend has also been observed in a recent benchmark for an unrelated set of molecules [122. In agreement with van Setten [27, we have found poles in the self-energy close to the predicted $\mathrm{QP}$ energies for $\mathrm{BN}, \mathrm{O}_{3}, \mathrm{BeO}, \mathrm{MgO}$ and $\mathrm{CuCN}$. Since analytic continuation has difficulties to resolve the precise pole structure of the Green's function, we only report the values obtained from the linearized self-energy.

Our discussion starts with the molecules that show large discrepancies between VASP and GTO's. A large out-liner is seemingly $\mathrm{CH}_{2} \mathrm{CHBr}$. However, for this molecule, as well as $\mathrm{C}_{6} \mathrm{H}_{5} \mathrm{OH}$, we found large forces in the preparatory PBE calculations. Double checking the original literature [123] suggests that the $G W 100$ paper used incorrect geometries. Since the ultimate purpose is certainly to compare with experiment, we decided to update the geometries to the correct literature values.

Among the remaining molecules, errors are large for compounds containing iodine, rubidium, and silver with a maximum deviation of $400 \mathrm{meV}$ for $\mathrm{CI}_{4}$ and $\mathrm{Rb}_{2}$, and $750 \mathrm{meV}$ for $\mathrm{Ag}_{2}$. However, in Ref. 27 no basis set extrapolation was performed for these molecules. From $\mathrm{CCl}_{4}$ to $\mathrm{CBr}_{4}$, the basis set corrections increase from 300 $\mathrm{meV}$ to $350 \mathrm{meV}$, suggesting a basis set error of $400 \mathrm{meV}$ for $\mathrm{CI}_{4}$ using GTOs. Similarly, for $\mathrm{Rb}_{2}$ the GTO results were not basis set corrected, and estimating the basis set error from $\mathrm{Na}_{2}$ and $\mathrm{K}_{2}$ again suggests that the VASP results are accurate. For $\mathrm{Ag}_{2}$, the difference between VASP and GTO seem on first sight to be too large to be ascribed to basis set errors alone. To resolve the issue, one of us (MvS) repeated the $\mathrm{Xe}, \mathrm{Rb}_{2}, \mathrm{I}_{2}, \mathrm{CH}_{2} \mathrm{CHI}, \mathrm{CI}_{4}$, $\mathrm{AlI}_{3}$, and $\mathrm{Ag}_{2}$ calculations using scalar relativistic corrections and frozen core SVP, TZVP and QZVP basis sets. This yielded basis set extrapolated values summarized in Tab. II certainly now in good to very good agreement with the VASP values.

For the remaining molecules, the mean absolute deviation between the two codes and thus two completely different basis sets is only $60 \mathrm{meV}$, if we also exclude $\mathrm{Cu}_{2}$. For $\mathrm{Cu}_{2}$, the fluorine containing compounds, $\mathrm{H}_{2} \mathrm{O}$, as well as Ne the ionization potentials (IPs) are smaller in VASP, which we will now show to be related to slight deficiencies in the PAW potentials. Copper, neon, and fluorine and, to a lesser extent, oxygen are particularly difficult to describe using a plane wave based approach, since the $3 d$ and $2 p$ electrons are strongly localized. To cope with this, the $\mathrm{Cu}, \mathrm{F}$ and $\mathrm{Ne}$ potentials are already the three smallest core and hardest potentials used in the present work. But still, the partial waves do not conserve the norm exactly, which results in errors, if an electron is scattered into a plane wave with very high kinetic energy 25. To determine this error, we performed calculations with norm-conserving (or almost norm-conserving) $G W$ potentials for the molecules $\mathrm{Cu}_{2}, \mathrm{~N}_{2}, \mathrm{~F}_{2}, \mathrm{CF}_{4}, \mathrm{HF}, \mathrm{BF}$, $\mathrm{SF}_{4}$ and $\mathrm{H}_{2} \mathrm{O}$ reported in Tab. III using the potentials Cu_Sv_GW_nc, N_h_GW,...,F_h_GW. Except for HF, the QP energies are clearly shifted towards higher binding energies in these calculations, and the discrepancies to the GTO calculations are reduced to an acceptable level of $100 \mathrm{meV}$. We also note that the PAW error increases from nitrogen, over oxygen to fluorine. $\mathrm{HF}$ and $\mathrm{BF}$ are exceptions, since the HOMOs possess predominantly hydrogen and boron character and, therefore, do not depend strongly on the $\mathrm{F}$ potential (we note that the $\mathrm{HF}$ results were already accurate using the standard potentials). Finally, the standard carbon and boron potentials used here are already almost norm conserving, and hence negligible changes are found for carbon based compounds with harder potentials (not shown).

The final case worthwhile mentioning is $\mathrm{KBr}$. Here the $G W 100$ paper 27] reports relatively large extrapolation errors of $130 \mathrm{meV}$, indicating that in this case the GTO 
TABLE I. Ionization potential (IP, negative of HOMO QP energies) for 100 molecules using $G_{0} W_{0}$ and linearized lin- $G_{0} W_{0}$ method. For comparison the basis set extrapolated values of Ref. 27 and the experimental IPs are given (vertical IPs are in italics). If basis set extrapolated values are not specified in Ref. 27, the AIMS-P16 values are shown in the column GTO $\left(\right.$ marked by $\left.{ }^{*}\right)$. Last column shows the differences between GTO and PW results. The ${ }^{*}$ indicates differences to non basis set extrapolated values.

\begin{tabular}{|c|c|c|c|c|c|c|}
\hline & & $\begin{array}{r}G_{0} W_{0} \\
\mathrm{GTO}[27\end{array}$ & $\begin{array}{r}G_{0} W_{0} \\
\mathrm{PW}\end{array}$ & $\begin{array}{r}\text { lin- } G_{0} W_{0} \\
\mathrm{PW}\end{array}$ & EXP & $\begin{array}{r}\Delta \\
\text { PW-GTO }\end{array}$ \\
\hline 1 & $\mathrm{He}$ & $23.49(0.03)$ & 23.38 & 23.62 & 24.5946 & -0.11 \\
\hline 2 & $\mathrm{Ne}$ & $20.33(0.01)$ & 20.17 & 20.36 & 21.56 [4] & -0.16 \\
\hline 3 & $\mathrm{Ar}$ & $15.28(0.03)$ & 15.32 & 15.42 & 15.76 [4] & 0.04 \\
\hline 4 & $\mathrm{Kr}$ & $13.89(0.16)$ & 13.93 & 14.03 & 14.0048 & 0.04 \\
\hline 5 & $\mathrm{Xe}$ & $12.02^{*}$ & 12.14 & 12.22 & 12.1349 & $0.12^{*}$ \\
\hline 6 & $\mathrm{H}_{2}$ & $15.85(0.09)$ & 15.85 & 16.06 & 15.4350 & 0.00 \\
\hline 7 & $\mathrm{Li}_{2}$ & $5.05(0.02)$ & 5.09 & 5.32 & 4.7351 & 0.04 \\
\hline 8 & $\mathrm{Na}_{2}$ & $4.88(0.03)$ & 4.93 & 5.06 & 4.8952 & 0.05 \\
\hline 9 & $\mathrm{Na}_{4}$ & $4.14(0.03)$ & 4.17 & 4.23 & 4.2753 & 0.03 \\
\hline 10 & $\mathrm{Na}_{6}$ & $4.34(0.06)$ & 4.34 & 4.40 & 4.1253 & 0.00 \\
\hline 11 & $\mathrm{~K}_{2}$ & $4.08(0.04)$ & 4.12 & 4.24 & 4.0652 & 0.04 \\
\hline 12 & $\mathrm{Rb}_{2}$ & $3.79^{*}$ & 4.02 & 4.14 & 3.9052 & $0.23^{*}$ \\
\hline 13 & $\mathrm{~N}_{2}$ & $15.05(0.04)$ & 14.93 & 15.06 & 15.5854 & -0.12 \\
\hline 14 & $\mathrm{P}_{2}$ & $10.38(0.04)$ & 10.35 & 10.40 & 10.6255 & -0.03 \\
\hline 15 & $\mathrm{As}_{2}$ & $9.67(0.10)$ & 9.59 & 9.62 & $10.0[56$ & -0.08 \\
\hline 16 & $\mathrm{~F}_{2}$ & $15.10(0.04)$ & 14.93 & 15.08 & 15.7057 & -0.17 \\
\hline 17 & $\mathrm{Cl}_{2}$ & $11.31(0.05)$ & 11.32 & 11.40 & $11.49[58$ & 0.01 \\
\hline 18 & $\mathrm{Br}_{2}$ & $10.56(0.18)$ & 10.57 & 10.65 & 10.5158 & 0.01 \\
\hline 19 & $\mathrm{I}_{2}$ & $9.23^{*}$ & 9.52 & 9.59 & 9.36 [59] & $0.29^{*}$ \\
\hline 20 & $\mathrm{CH}_{4}$ & $14.00(0.06)$ & 14.02 & 14.14 & 13.660 & 0.02 \\
\hline 21 & $\mathrm{C}_{2} \mathrm{H}_{6}$ & $12.46(0.06)$ & 12.50 & 12.58 & 11.9959 & 0.04 \\
\hline 22 & $\mathrm{C}_{3} \mathrm{H}_{8}$ & $11.89(0.06)$ & 11.90 & 11.98 & 11.5159 & 0.01 \\
\hline 23 & $\mathrm{C}_{4} \mathrm{H}_{10}$ & $11.59(0.05)$ & 11.61 & 11.69 & 11.0959 & 0.02 \\
\hline 24 & $\mathrm{C}_{2} \mathrm{H}_{4}$ & $10.40(0.03)$ & 10.42 & 10.50 & 10.6860 & 0.02 \\
\hline 25 & $\mathrm{C}_{2} \mathrm{H}_{2}$ & $11.09(0.01)$ & 11.07 & 11.24 & 11.4960 & -0.02 \\
\hline 26 & $\mathrm{C}_{4}$ & $10.91(0.03)$ & 10.89 & 10.97 & 12.5461 & -0.02 \\
\hline 27 & $\mathrm{C}_{3} \mathrm{H}_{6}$ & $10.65(0.04)$ & 10.72 & 10.78 & 10.5462 & 0.07 \\
\hline 28 & $\mathrm{C}_{6} \mathrm{H}_{6}$ & $9.10(0.01)$ & 9.11 & 9.16 & 9.2363 & 0.01 \\
\hline 29 & $\mathrm{C}_{8} \mathrm{H}_{8}$ & $8.18(0.02)$ & 8.19 & 8.24 & 8.4364 & 0.01 \\
\hline 30 & $\mathrm{C}_{5} \mathrm{H}_{6}$ & $8.45(0.02)$ & 8.47 & 8.51 & 8.5365 & 0.02 \\
\hline 31 & $\mathrm{CH}_{2} \mathrm{CHF}$ & $10.32(0.02)$ & 10.28 & 10.36 & 10.6366 & -0.04 \\
\hline 32 & $\mathrm{CH}_{2} \mathrm{CHCl}$ & $9.89(0.02)$ & 9.92 & 10.00 & 10.2067 & 0.03 \\
\hline 33 & $\mathrm{CH}_{2} \mathrm{CHBr}$ & $9.14(0.01)$ & 9.75 & 9.83 & 9.9067 & 0.61 \\
\hline 34 & $\mathrm{CH}_{2} \mathrm{CHI}$ & $9.01^{*}$ & 9.27 & 9.36 & 9.3568 & $0.26^{*}$ \\
\hline 35 & $\mathrm{CF}_{4}$ & $15.60(0.06)$ & 15.41 & 15.53 & 16.2069 & -0.19 \\
\hline 36 & $\mathrm{CCl}_{4}$ & $11.21(0.06)$ & 11.20 & 11.31 & $11.69[59$ & -0.01 \\
\hline 37 & $\mathrm{CBr}_{4}$ & $10.22(0.16)$ & 10.25 & 10.38 & 10.5470 & 0.03 \\
\hline 38 & $\mathrm{CI}_{4}$ & $8.71^{*}$ & 9.11 & 9.23 & $9.10 \square 1$ & $0.40^{*}$ \\
\hline 39 & $\mathrm{SiH}_{4}$ & $12.40(0.06)$ & 12.40 & 12.53 & 12.372 & 0.00 \\
\hline 40 & $\mathrm{GeH}_{4}$ & $12.11(0.04)$ & 12.13 & 12.24 & 11.3473 & 0.02 \\
\hline 41 & $\mathrm{H}_{6} \mathrm{Si}_{2}$ & $10.41(0.06)$ & 10.44 & 10.52 & 10.5374 & 0.03 \\
\hline 42 & $\mathrm{H}_{12} \mathrm{Si}_{5}$ & $9.05(0.05)$ & 9.13 & 9.19 & 9.3674 & 0.08 \\
\hline 43 & $\mathrm{LiH}$ & $6.58(0.04)$ & 6.46 & 7.20 & 7.9075 & -0.12 \\
\hline 44 & $\mathrm{KH}$ & $4.99(0.01)$ & 4.97 & 5.37 & 8.0076 & -0.02 \\
\hline 45 & $\mathrm{BH}_{3}$ & $12.96(0.06)$ & 12.95 & 13.09 & 12.0377 & -0.01 \\
\hline 46 & $\mathrm{~B}_{2} \mathrm{H}_{6}$ & $11.93(0.06)$ & 11.94 & 12.04 & 11.9078 & 0.01 \\
\hline 47 & $\mathrm{NH}_{3}$ & $10.39(0.05)$ & 10.32 & 10.44 & 10.8279 & -0.07 \\
\hline 48 & $\mathrm{HN}_{3}$ & $10.55(0.02)$ & 10.50 & 10.56 & 10.7280 & -0.05 \\
\hline
\end{tabular}




\begin{tabular}{|c|c|c|c|c|c|c|}
\hline & & $\begin{array}{r}G_{0} W_{0} \\
\text { GTO }\end{array}$ & $\begin{array}{r}G_{0} W_{0} \\
\mathrm{PW}\end{array}$ & $\begin{array}{r}\text { lin- } G_{0} W_{0} \\
\mathrm{PW}\end{array}$ & EXP & $\begin{array}{r}\Delta \\
\text { PW-GTO } \\
\end{array}$ \\
\hline 49 & $\mathrm{PH}_{3}$ & $10.35(0.05)$ & 10.35 & 10.45 & 10.5981 & 0.00 \\
\hline 50 & $\mathrm{AsH}_{3}$ & $10.21(0.02)$ & 10.26 & 10.36 & $10.58[82]$ & 0.05 \\
\hline 51 & $\mathrm{H}_{2} \mathrm{~S}$ & $10.13(0.04)$ & 10.11 & 10.30 & 10.5083 & -0.02 \\
\hline 52 & $\mathrm{HF}$ & $15.37(0.01)$ & 15.37 & 15.38 & 16.12 [84] & 0.00 \\
\hline 53 & $\mathrm{HCl}$ & $12.36(0.01)$ & 12.45 & 12.51 & 12.7985 & 0.09 \\
\hline 54 & $\mathrm{LiF}$ & $10.27(0.03)$ & 10.07 & 10.45 & 11.30 [86] & -0.20 \\
\hline 55 & $\mathrm{MgF}_{2}$ & $12.50(0.06)$ & 12.41 & 12.77 & 13.30 [87] & -0.09 \\
\hline 56 & $\mathrm{TiF}_{4}$ & $14.07(0.05)$ & 14.01 & 14.22 & 15.3088 & -0.06 \\
\hline 57 & $\mathrm{AlF}_{3}$ & $14.48(0.06)$ & 14.33 & 14.53 & 15.4589 & -0.15 \\
\hline 58 & $\mathrm{BF}$ & $10.73(0.05)$ & 10.46 & 10.67 & 11.00 90] & -0.27 \\
\hline 59 & $\mathrm{SF}_{4}$ & $12.38(0.07)$ & 12.20 & 12.29 & 11.69 [91] & -0.18 \\
\hline 60 & $\mathrm{KBr}$ & $7.57(0.13)$ & 7.80 & 8.04 & 8.8292 & 0.23 \\
\hline 61 & $\mathrm{GaCl}$ & $9.74(0.07)$ & 9.89 & 9.99 & 10.0793 & 0.15 \\
\hline 62 & $\mathrm{NaCl}$ & $8.43(0.14)$ & 8.47 & 8.76 & 9.8092 & 0.04 \\
\hline 63 & $\mathrm{MgCl}_{2}$ & $11.20(0.07)$ & 11.19 & 11.41 & 11.80 94] & -0.01 \\
\hline 64 & $\mathrm{AlI}_{3}$ & $9.30^{*}$ & 9.58 & 9.69 & 9.66 [95] & $0.28^{*}$ \\
\hline 65 & $\mathrm{BN}$ & $11.15(0.03)$ & - & 10.61 & 11.50 & - \\
\hline 66 & $\mathrm{HCN}$ & $13.32(0.01)$ & 13.29 & 13.43 & $13.61[96]$ & -0.03 \\
\hline 67 & $\mathrm{PN}$ & $11.29(0.04)$ & 11.24 & 11.41 & 11.8897 & -0.05 \\
\hline 68 & $\mathrm{~N}_{2} \mathrm{H}_{4}$ & $9.37(0.04)$ & 9.33 & 9.45 & 8.9898 & -0.04 \\
\hline 69 & $\mathrm{H}_{2} \mathrm{CO}$ & $10.46(0.02)$ & 10.42 & 10.57 & 10.88 [9] & -0.04 \\
\hline 70 & $\mathrm{CH}_{3} \mathrm{OH}$ & $10.67(0.05)$ & 10.61 & 10.72 & $10.96[100]$ & -0.06 \\
\hline 71 & $\mathrm{CH}_{3} \mathrm{CH}_{2} \mathrm{OH}$ & $10.27(0.05)$ & 10.21 & 10.33 & 10.64 [101] & -0.06 \\
\hline 72 & $\mathrm{CH}_{3} \mathrm{CHO}$ & $9.66(0.03)$ & 9.63 & 9.80 & $10.24[102$ & -0.03 \\
\hline 73 & $\mathrm{CH}_{3} \mathrm{CH}_{2} \mathrm{OCH}_{2} \mathrm{CH}_{3}$ & $9.42(0.05)$ & 9.43 & 9.52 & 9.61 [101] & 0.01 \\
\hline 74 & $\mathrm{HCOOH}$ & $10.87(0.01)$ & 10.81 & 10.98 & 11.50 [103] & -0.06 \\
\hline 75 & $\mathrm{H}_{2} \mathrm{O}_{2}$ & $11.10(0.01)$ & 10.96 & 11.12 & $11.70[104$ & -0.14 \\
\hline 76 & $\mathrm{H}_{2} \mathrm{O}$ & $12.05(0.03)$ & 11.84 & 12.05 & 12.62 [59] & -0.21 \\
\hline 77 & $\mathrm{CO}_{2}$ & $13.46(0.06)$ & 13.36 & 13.44 & 13.77 [105] & -0.10 \\
\hline 78 & $\mathrm{CS}_{2}$ & $9.95(0.05)$ & 9.96 & 10.01 & 10.09 [106] & 0.01 \\
\hline 79 & $\mathrm{CSO}$ & $11.11(0.05)$ & 11.06 & 11.13 & 11.19 [107] & -0.05 \\
\hline 80 & COSe & $10.43(0.09)$ & 10.42 & 10.50 & 10.37 [108] & -0.01 \\
\hline 81 & $\mathrm{CO}$ & $13.71(0.04)$ & 13.62 & 13.76 & 14.01 [107] & -0.09 \\
\hline 82 & $\mathrm{O}_{3}$ & $11.49(0.03)$ & - & 12.07 & 12.73 [109] & - \\
\hline 83 & $\mathrm{SO}_{2}$ & $12.06(0.06)$ & 11.91 & 12.04 & 12.50 [59] & -0.15 \\
\hline 84 & $\mathrm{BeO}$ & $8.60(0.01)$ & - & 9.50 & 10.10 110 & - \\
\hline 85 & $\mathrm{MgO}$ & $6.75(0.03)$ & - & 7.10 & 8.76 [111] & - \\
\hline 86 & $\mathrm{C}_{6} \mathrm{H}_{5} \mathrm{CH}_{3}$ & $8.73(0.02)$ & 8.75 & 8.79 & $8.82[63$ & 0.02 \\
\hline 87 & $\mathrm{C}_{8} \mathrm{H}_{10}$ & $8.66(0.02)$ & 8.69 & 8.73 & 8.7763 & 0.03 \\
\hline 88 & $\mathrm{C}_{6} \mathrm{~F}_{6}$ & $9.74(0.07)$ & 9.63 & 9.69 & 10.2069 & -0.11 \\
\hline 89 & $\mathrm{C}_{6} \mathrm{H}_{5} \mathrm{OH}$ & $8.51(0.01)$ & 8.38 & 8.43 & $8.75[112$ & -0.13 \\
\hline 90 & $\mathrm{C}_{6} \mathrm{H}_{5} \mathrm{NH}_{2}$ & $7.78(0.01)$ & 7.78 & 7.84 & 8.05 [113] & 0.00 \\
\hline 91 & $\mathrm{C}_{5} \mathrm{H}_{5} \mathrm{~N}$ & $9.17(0.01)$ & 9.16 & 9.31 & 9.66 [114] & -0.01 \\
\hline 92 & Guanine & $7.87(0.01)$ & 7.85 & 7.90 & $8.24[115$ & -0.02 \\
\hline 93 & Adenine & $8.16(0.01)$ & 8.12 & 8.18 & 8.48 [116] & -0.04 \\
\hline 94 & Cytosine & $8.44(0.01)$ & 8.40 & 8.50 & 8.94 [115] & -0.04 \\
\hline 95 & Thymine & $8.87(0.01)$ & 8.83 & 8.89 & 9.20 [117] & -0.04 \\
\hline 96 & Uracil & $9.38(0.01)$ & 9.36 & 9.55 & 9.68 [118] & -0.02 \\
\hline 97 & $\mathrm{NH}_{2} \mathrm{CONH}_{2}$ & $9.46(0.02)$ & 9.35 & 9.59 & 9.8083 & -0.11 \\
\hline 98 & $\mathrm{Ag}_{2}$ & $7.08^{*}$ & 7.83 & 7.95 & 7.66 119 & $0.75^{*}$ \\
\hline 99 & $\mathrm{Cu}_{2}$ & $7.78(0.06)$ & 7.19 & 7.40 & 7.46 [120] & -0.59 \\
\hline 100 & $\mathrm{CuCN}$ & $9.56(0.04)$ & - & 9.99 & - & - \\
\hline
\end{tabular}


TABLE II. IP (negative HOMO $G_{0} W_{0} \mathrm{QP}$ energies) and $G_{0} W_{0}$ LUMO for selected molecules. The GTO values have been calculated using frozen core potentials, scalar relativistic corrections, and are extrapolated to the infinite basis set limit.

\begin{tabular}{llrrrr}
\hline \hline & & IP & IP & LUMO & LUMO \\
& & GTO & PW & GTO & PW \\
\hline 5 & $\mathrm{Xe}$ & 12.22 & 12.14 & -0.07 & 0.28 \\
12 & $\mathrm{Rb}_{2}$ & 4.07 & 4.02 & -0.85 & -0.74 \\
19 & $\mathrm{I}_{2}$ & 9.48 & 9.52 & -2.28 & -2.21 \\
34 & $\mathrm{CH}_{2} \mathrm{CHI}$ & 9.13 & 9.27 & 0.56 & 0.37 \\
38 & $\mathrm{CI}_{4}$ & 8.97 & 9.11 & -2.47 & -2.42 \\
64 & $\mathrm{AlI}_{3}$ & 9.50 & 9.58 & -1.18 & -1.02 \\
98 & $\mathrm{Ag}_{2}$ & 7.96 & 7.83 & -1.40 & -1.35 \\
\hline \hline
\end{tabular}

TABLE III. IP (negative HOMO $G_{0} W_{0}$ QP energies) for selected molecules calculated for a $9 \AA$ box for the standard $G W$ potentials and normconserving $G W$ potentials. Results differ from the previous table, since calculations in Table II have been performed for larger boxes and include a correction for the box size error. The column $\Delta$ reports the difference between the standard PAW and NC PAW potential.

\begin{tabular}{llrrr}
\hline \hline & & $G W$ PAW & NC $G W$ PAW & $\Delta$ \\
\hline 13 & $\mathrm{~N}_{2}$ & 14.98 & 15.02 & -0.04 \\
16 & $\mathrm{~F}_{2}$ & 14.97 & 15.13 & -0.15 \\
35 & $\mathrm{CF}_{4}$ & 15.42 & 15.58 & -0.16 \\
52 & $\mathrm{HF}$ & 15.39 & 15.32 & 0.06 \\
58 & $\mathrm{BF}$ & 10.42 & 10.46 & -0.04 \\
59 & $\mathrm{SF}_{4}$ & 12.19 & 12.26 & -0.07 \\
76 & $\mathrm{H}_{2} \mathrm{O}$ & 11.86 & 11.94 & -0.09 \\
99 & $\mathrm{Cu}_{2}$ & 7.03 & 7.53 & -0.50 \\
\hline \hline
\end{tabular}

based extrapolation might be inaccurate.

For the remaining systems, we find the agreement to be excellent. Specifically, for all considered organic molecules the absolute differences are typically below 50 meV, with very few out-liners. This clearly demonstrates that plane wave codes can be competitive in terms of precision with GTOs. Certainly the agreement between GTOs and PWs is better than originally reported in the $G W 100$ paper, a point discussed in more detail in the next section.

\section{B. Basis set convergence and comparison to other PW calculations}

In Fig. 1, we show the convergence of the HOMO with respect to the plane wave cutoff for the orbitals. This cutoff also determines the total number of orbitals as well as the cutoff for the response function. The number of plane waves and total orbitals is proportional to one over the cutoff to the power of $3 / 2$. It is clearly visible

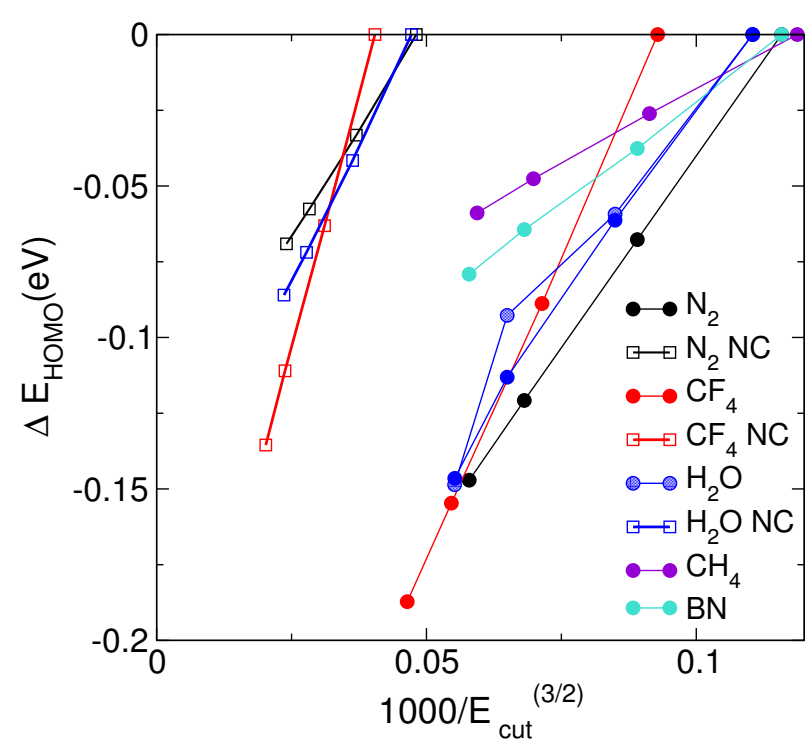

FIG. 1. (color online) Convergence of QP HOMO with respect to the employed cutoff for various materials. For $\mathrm{N}_{2}$, $\mathrm{CF}_{4}$ and $\mathrm{H}_{2} \mathrm{O}$, results are shown for two potentials, the standard $G W$ potentials, as well as NC potentials. The slopes are steeper for the NC potentials, which is particularly obvious for $\mathrm{CF}_{4}$. For $\mathrm{H}_{2} \mathrm{O}$ results for two box sizes ( 8 and $9 \AA$ ) are shown (see text).

that the curves follow almost exactly a straight line. In few cases, out-liners are visible. For instance for $\mathrm{H}_{2} \mathrm{O}$, we have included results for two box sizes 8 and $9 \AA$. The $9 \AA$ box results have a slight jump, which is not present for the $8 \AA$ box. However, this out-liner is small (about $10 \mathrm{meV}$ ), and changes extrapolated results only by less than $10 \mathrm{meV}$. Usually the out-liners could be dealt with by just changing the box size slightly. We believe that they are related to "shell"-effects, i.e. a sudden increase in the number of plane waves when the cutoff is changed through certain values. Furthermore, the analytic continuation is not always entirely well behaved and can cause changes of the order of $20 \mathrm{meV}$. Overall, the plot demonstrates that extrapolation with respect to the energy-cutoff is very well under control and can be done with great accuracy.

As noted before, the differences between the GTO and the Berkeley $G W$ calculations reported in Ref. 27 are more sizable. If we exclude the difficult multipole cases, $\mathrm{BN}, \mathrm{O}_{3}, \mathrm{MgO}$ and $\mathrm{BeO}$, the mean absolute deviation between Berkeley $G W$ and basis set extrapolated GTOs was $0.2 \mathrm{eV}$ in Ref. 27, whereas it is reduced to $0.05 \mathrm{eV}$ for VASP PAW potentials (for the same subset). We speculate that this is mostly related to neglecting basis set extrapolation errors or - less likely - to an inaccurate treatment of the core-valence interaction. Typically our basis set corrections are of the order of 300-400 meV at the default cutoff and therefore very sizable. Even doubling the number of basis functions and therefore increasing the compute time by a factor of about 8 (cubic 
scaling), reduces the error only by a factor 2 , to about 150-200 meV. Hence, calculations without basis set corrections are hardly affordable or practicable, and it is certainly advisable to perform an extrapolation whenever possible.

For the core-valence interaction, we emphasize that VASP always evaluates the interaction at the level of Hartree-Fock if correlated calculations are performed. More precisely, VASP calculates the PBE core orbitals on the fly and then recalculates the action of the PBE core states on the valence states using the Hartree-Fock approximation. Not doing so can have a sizable effect on the QP energies for heavier atoms 34]. We are not aware of other pseudopotential codes following a similar route. This might be responsible for a small part of the errors in the reported Berkeley $G W$ calculations of Ref. 27, if heavier atoms are involved.

Calculations for another fairly large set of molecules have been reported by Govoni and Galli using the West code[24]. 29 molecules are identical to the $G W 100$ set considered here. The mean absolute difference between the basis set extrapolated GTO results and the VASP results for this subset is $60 \mathrm{meV}$, whereas the difference between the West results and the basis set extrapolated GTO results is about twice as large $120 \mathrm{meV}$ (mean absolute difference between VASP and West is $90 \mathrm{meV}$ ). In many cases, the West IPs are too small indicating again basis set incompleteness errors. Anyhow, the West results are closer to the basis set converged values than the $G W$ Berkeley results.

\section{Comparison to experiment}

When comparing the present results against the experimental ionisation energies, a mean absolute error of $0.5 \mathrm{eV}$ is observed. This large discrepancy is not unexpected given that in this computational approach selfconsistency, vertex corrections and finite temperature effects are omitted. However we can comment on the biggest outliers in the set. A first example is $\mathrm{C}_{4}$ : it is well known that the smaller $\mathrm{C}_{2}$ molecule is particularly challenging to describe, owing to strong electron correlation [144]. For the larger cluster we expect similar effects, hence the inclusion of the vertex should improve the agreement with the experiment. We have a similar expectation for the case of $\mathrm{F}_{2}$. Our conjecture is substantiated by previous electron propagator calculations [145], where the poles of the Green's function in the Lehmann representation were located to give the IP, and where a comparable mismatch to experiment was ascribed to the poor description of dynamic correlation. For $\mathrm{AlF}_{3}, \mathrm{LiF}$ and $\mathrm{KH}$ we have to bear in mind that the experimental value for a vertical transition was not available, therefore geometry relaxations may explain the mismatch. This is only partially true for $\mathrm{KH}$, where the inclusion of adiabatic effects in the perturbative calculations still leaves a sizable disagreement $(\sim 2 \mathrm{eV})[146$; in this case it is not completely unreasonable to call for a further assessment of the experimental value.

\section{Linearized QP-HOMO for $G W \mathbf{1 0 0}$}

We now turn to results obtained by first linearizing the self-energy and then determining the QP energy from this linearized equation. This procedure is in our experience more "robust" and better behaved than seeking the poles in the non-linearized equation. The main issue of the latter approach is that, in the $G_{0} W_{0}$ approximation, the first pole in the self-energy is approximately located at the energy of the DFT HOMO minus the first excitation energy in the DFT (LUMO-HOMO):

$$
\epsilon_{\mathrm{HOMO}}-\left(\epsilon_{\mathrm{LUMO}}-\epsilon_{\mathrm{HOMO}}\right)
$$

This is a simple Auger like excitation, where the hole has sufficient energy, i.e. is sufficiently below the HOMO to be able to excite an electron-hole pair. As discussed by van Setten, such poles lead to multiple solutions for the QP energy 27] and make the determination of the QP energies difficult for molecules with small excitation energies. These poles are, however, an artifact of the $G_{0} W_{0}$ approximation. If the $G W$ procedure were done self-consistently, the first pole in the self-energy would move to approximately

$$
E_{\mathrm{HOMO}}^{\mathrm{QP}}-\left(E_{\mathrm{LUMO}}^{\mathrm{QP}}-E_{\mathrm{HOMO}}^{\mathrm{QP}}\right)
$$

In other words, at the valence band edge (HOMO) and conduction band edge (LUMO) the self-energy never possesses poles. However, in a single shot procedure and when starting from much too small band gaps, the quasiparticle energy $E^{\mathrm{QP}}$ might move into regions where the self-energy evaluated from DFT orbitals has a pole. Linearization at the DFT eigenenergies resolves this issue, as the $G_{0} W_{0}$ self-energy has no poles in the direct vicinity of the DFT HOMO. The problem is also less severe, if the calculations are done selfconsistently or when starting from a prescription that yields larger HOMO-LUMO Kohn-Sham gaps, as shown in a recent evaluation of the difference between the quasi-particle orbital energies and their linearized counterparts by Govoni et al. [24]. Therein it is shown that, for a wide range of molecules, this difference is substantially more pronounced for $G W$ calculations on a PBE reference state than if a hybrid functional with non-local exchange is used.

In summary, we feel that for code benchmarking as well as for a comparison with experiment determining the poles of the linearized equation is preferable, at least, if a PBE reference state is employed. However, it also needs to be emphasized that for comparison with the already published $G W 100$ data, it is of paramount importance to follow exactly the procedures laid out in the the initial $G W 100$ paper. 
TABLE IV. LUMO QP energies for selected molecules using $G_{0} W_{0}$ and the linearized lin- $G_{0} W_{0}$. For comparison, the non basis set extrapolated values (AIMS-P16), the basis set extrapolated values from Ref. 27, and the negative of the experimental electron affinities are shown (vertical attachment energies are in italics). Differences between PW and GTO are shown in last column. The ${ }^{+}$indicates energies above the vacuum level, and ${ }^{*}$ indicates differences to non basis set extrapolated values.

\begin{tabular}{|c|c|c|c|c|c|c|c|}
\hline & & $\begin{array}{r}G_{0} W_{0} \\
\text { AIMS-P16 }\end{array}$ & $\begin{array}{r}G_{0} W_{0} \\
\text { GTO-EXTRA } \\
\end{array}$ & $\begin{array}{r}G_{0} W_{0} \\
\mathrm{PW}\end{array}$ & $\begin{array}{r}\operatorname{lin}-G_{0} W_{0} \\
\mathrm{PW} \\
\end{array}$ & EXP & $\begin{array}{r}\Delta \\
\text { PW-GTO }\end{array}$ \\
\hline 2 & $\mathrm{Ne}$ & 11.64 & - & 0.40 & 0.40 & - & $-11.24^{+}$ \\
\hline 5 & $\mathrm{Xe}$ & 4.28 & - & 0.70 & 0.70 & - & $-3.58^{+}$ \\
\hline 6 & $\mathrm{H}_{2}$ & 3.50 & $3.30(0.52)$ & 0.07 & 0.07 & - & $-3.23^{+}$ \\
\hline 7 & $\mathrm{Li}_{2}$ & -0.63 & $-0.75(0.04)$ & -0.61 & -0.54 & - & 0.14 \\
\hline 8 & $\mathrm{Na}_{2}$ & -0.55 & $-0.66(0.70)$ & -0.60 & -0.56 & -0.54 & 0.06 \\
\hline 9 & $\mathrm{Na}_{4}$ & -1.01 & $-1.15(0.90)$ & -1.07 & -1.03 & -0.91124 & 0.08 \\
\hline 10 & $\mathrm{Na}_{6}$ & -0.97 & $-1.13(0.10)$ & -1.07 & -1.03 & - & 0.06 \\
\hline 11 & $\mathrm{~K}_{2}$ & -0.65 & $-0.75(0.05)$ & -0.74 & -0.70 & -0.50 & 0.01 \\
\hline 12 & $\mathrm{Rb}_{2}$ & -0.62 & - & -0.74 & -0.70 & -0.50124 & $-0.12^{*}$ \\
\hline 14 & $\mathrm{P}_{2}$ & -0.72 & $-1.08(0.08)$ & -0.99 & -0.97 & -0.68 [125 & 0.09 \\
\hline 15 & $\mathrm{As}_{2}$ & -0.85 & $-1.52(0.35)$ & -1.07 & -1.06 & -0.74126 & 0.45 \\
\hline 16 & $\mathrm{~F}_{2}$ & -0.70 & $-1.23(0.14)$ & -0.96 & -0.84 & $-1.24[127]$ & 0.27 \\
\hline 17 & $\mathrm{Cl}_{2}$ & -0.89 & $-1.40(0.12)$ & -1.25 & -1.22 & -1.02 127 & 0.15 \\
\hline 18 & $\mathrm{Br}_{2}$ & -1.40 & $-1.96(0.29)$ & -1.99 & -1.97 & -1.60 [127] & -0.03 \\
\hline 19 & $\mathrm{I}_{2}$ & -1.68 & - & -2.21 & -2.20 & -1.70127 & $-0.53^{*}$ \\
\hline 20 & $\mathrm{CH}_{4}$ & 2.45 & $2.03(0.35)$ & 0.63 & 0.63 & - & $-1.40^{+}$ \\
\hline 26 & $\mathrm{C}_{4}$ & -2.94 & $-3.15(0.06)$ & -3.09 & -3.08 & -3.88128 & 0.06 \\
\hline 29 & $\mathrm{C}_{8} \mathrm{H}_{8}$ & 0.06 & $-0.12(0.02)$ & -0.05 & -0.02 & -0.57 [129] & 0.07 \\
\hline 32 & $\mathrm{CH}_{2} \mathrm{CHCl}$ & 1.42 & $1.17(0.03)$ & 1.19 & 1.25 & - & $0.02^{+}$ \\
\hline 36 & $\mathrm{CCl}_{4}$ & -0.01 & $-0.54(0.13)$ & -0.32 & -0.28 & - & 0.22 \\
\hline 37 & $\mathrm{CBr}_{4}$ & -1.08 & $-1.56(0.29)$ & -1.47 & -1.44 & - & 0.09 \\
\hline 38 & $\mathrm{CI}_{4}$ & -2.14 & - & -2.42 & -2.40 & - & $-0.28^{*}$ \\
\hline 42 & $\mathrm{H}_{12} \mathrm{Si}_{5}$ & 0.16 & $0.00(0.07)$ & 0.03 & 0.05 & - & 0.03 \\
\hline 43 & $\mathrm{LiH}$ & -0.07 & $-0.16(0.09)$ & -0.07 & -0.04 & -0.34 [130 & 0.09 \\
\hline 44 & $\mathrm{KH}$ & -0.18 & $-0.32(0.01)$ & -0.25 & -0.22 & - & 0.07 \\
\hline 45 & $\mathrm{BH}_{3}$ & 0.12 & $0.03(0.05)$ & 0.03 & 0.08 & -0.04131 & 0.00 \\
\hline 54 & $\mathrm{LiF}$ & 0.09 & $-0.01(0.01)$ & 0.17 & 0.17 & - & 0.18 \\
\hline 55 & $\mathrm{MgF}_{2}$ & -0.14 & $-0.31(0.06)$ & -0.29 & -0.28 & - & 0.02 \\
\hline 56 & $\mathrm{TiF}_{4}$ & -0.60 & $-1.06(0.13)$ & -0.79 & -0.66 & -2.50132 & 0.27 \\
\hline 57 & $\mathrm{AlF}_{3}$ & 0.16 & $-0.23(0.10)$ & 0.08 & 0.09 & - & 0.31 \\
\hline 59 & $\mathrm{SF}_{4}$ & 0.38 & $-0.10(0.13)$ & 0.07 & 0.12 & -1.50133 & 0.17 \\
\hline 60 & $\mathrm{KBr}$ & -0.31 & $-0.42(0.06)$ & -0.32 & -0.31 & -0.64 [134 & 0.10 \\
\hline 61 & $\mathrm{GaCl}$ & -0.02 & $-0.39(0.15)$ & -0.19 & -0.15 & - & 0.20 \\
\hline 62 & $\mathrm{NaCl}$ & -0.39 & $-0.42(0.01)$ & -0.46 & -0.43 & -0.73134 & -0.04 \\
\hline 63 & $\mathrm{MgCl}_{2}$ & -0.43 & $-0.68(0.08)$ & -0.61 & -0.59 & - & 0.07 \\
\hline 64 & $\mathrm{AlI}_{3}$ & -0.80 & - & -1.02 & -0.99 & - & $-0.22^{*}$ \\
\hline 72 & $\mathrm{CH}_{3} \mathrm{CHO}$ & 1.05 & $0.83(0.05)$ & 0.87 & 0.87 & - & $0.04^{+}$ \\
\hline 74 & $\mathrm{HCOOH}$ & 1.91 & $1.59(0.00)$ & 1.64 & 1.72 & - & $0.05^{+}$ \\
\hline 76 & $\mathrm{H}_{2} \mathrm{O}$ & 2.37 & $2.01(0.16)$ & 1.04 & 1.04 & - & $-0.97^{+}$ \\
\hline 78 & $\mathrm{CS}_{2}$ & -0.20 & $-0.55(0.09)$ & -0.42 & -0.40 & -0.55135 & 0.13 \\
\hline 82 & $\mathrm{O}_{3}$ & -2.30 & $-2.69(0.11)$ & -2.50 & -2.52 & -2.10 [136 & 0.19 \\
\hline 83 & $\mathrm{SO}_{2}$ & -1.00 & $-1.49(0.12)$ & -1.25 & -1.19 & -1.11137 & 0.24 \\
\hline 84 & $\mathrm{BeO}$ & -2.56 & $-2.72(0.04)$ & -2.73 & -2.37 & - & -0.01 \\
\hline 85 & $\mathrm{MgO}$ & -1.89 & $-2.13(0.09)$ & -2.05 & -2.12 & - & 0.08 \\
\hline 88 & $\mathrm{C}_{6} \mathrm{~F}_{6}$ & 0.66 & $0.36(0.08)$ & 0.24 & 0.27 & -0.70138 & $-0.12^{+}$ \\
\hline 94 & Cytosine & 0.26 & $0.01(0.01)$ & 0.12 & 0.15 & -0.23139 & 0.11 \\
\hline 95 & Thymine & 0.06 & $-0.18(0.01)$ & -0.06 & -0.04 & 0.29140 & 0.12 \\
\hline 96 & Uracil & 0.01 & $-0.25(0.01)$ & -0.11 & -0.09 & 0.22 140] & 0.14 \\
\hline 98 & $\mathrm{Ag}_{2}$ & -1.05 & - & -1.35 & -1.31 & -1.10141 & $-0.30^{*}$ \\
\hline 99 & $\mathrm{Cu}_{2}$ & -0.92 & $-1.23(0.08)$ & -1.24 & -1.21 & -0.84142 & -0.01 \\
\hline 100 & $\mathrm{CuCN}$ & -1.65 & $-1.85(0.05)$ & -1.91 & -1.81 & -1.47143 & -0.06 \\
\hline
\end{tabular}




\section{E. LUMO for $G W 100$}

The calculated LUMOs are shown in Table IV A few important comments are in place here. First, the table reports the QP energy of the lowest unoccupied orbital in the preceeding DFT calculations to maintain compatibility with the previous publication. In some cases (Xe, $\mathrm{H}_{2} \mathrm{O}, \mathrm{CH}_{2} \mathrm{CHCl}, \mathrm{CH}_{3} \mathrm{CHO}$, and $\left.\mathrm{HCOOH}\right) \mathrm{PW}$ calculations predict at the DFT level a very weakly bound LUMO+1 state (just below the vacuum level) whose $G_{0} W_{0} \mathrm{QP}$ energy is below the QP state corresponding to the DFT LUMO level. These energy levels are not shown in Tab. IV.

If we consider the $G_{0} W_{0}$ values corresponding to the DFT LUMOs, the agreement between the $G W 100$ reference GTO data and plane waves is reasonable, although not quite as good as for the HOMO. Specifically troublesome is the observation that the GTO calculations sometimes predict much too positive LUMOs several eV above the PW results. Admittedly, box size convergence can be troublesome for QP energies above the vacuum level, and we therefore only show few selected positive LUMOsthose where we are confident that convergence to $50 \mathrm{meV}$ was attained for the cell sizes considered in our calculations. All positive (unbound) $G_{0} W_{0}$ LUMOs are marked by a superscript " + sign in the last column. The differences are particularly striking for $\mathrm{Ne}, \mathrm{Xe}, \mathrm{H}_{2}, \mathrm{H}_{2} \mathrm{O}$ and $\mathrm{CH}_{4}$ reaching $11 \mathrm{eV}$ for $\mathrm{Ne}$.

To investigate this issue, we compared the DFTLUMOs of the PW and GTO calculations (the latter are available upon request to $\mathrm{MvS}$ ), and found that the deviations between PWs and GTOs are much larger than for the DFT HOMOs on average, and especially large for some of the problematic cases, e.g. $\mathrm{Ne}, \mathrm{Xe}, \mathrm{H}_{2}$, or $\mathrm{H}_{2} \mathrm{O}$ which are the largest outliners in the subsequent QP calculations. Specifically, for $\mathrm{Ne}$ and $\mathrm{H}_{2}$ the $\mathrm{PW}$ DFT calculations predict very shallow bound LUMOs, a few $10 \mathrm{meV}$ below the vacuum level. These can not be reproduced with any of the available GTO basis sets.

For the other cases with larger discrepencies, we now show that the GTO basis sets are often not sufficiently flexible to describe unoccupied orbitals. This is supported by several observations. (i) Basis set corrections using GTOs are much larger for the LUMO than for the HOMO, as for instance exemplified for $\mathrm{As}_{2}, \mathrm{~F}_{2}$ or $\mathrm{Cl}_{2}$. To make this very clear, we have included in Table IV both, the basis set extrapolated values (with estimated error bars), as well as the values at the largest considered GTO basis set. (ii) Non basis set extrapolated GTO values deviate markedly from PW results. As before, these are marked by a star superscript in the last column. GTO basis set extrapolated values are tabulated in Tab. II and clearly improve the agreement with the PW results. For $\mathrm{Xe}$, where the discrepancy was previously $3.6 \mathrm{eV}$, the error is reduced to about $0.3 \mathrm{eV}$. Furthermore, we recalculated the QP energies of $\mathrm{H}_{2} \mathrm{O}$ and $\mathrm{CH}_{4}$ using Dunning correlation consistent basis sets and found basis set extrapolated $G_{0} W_{0} \mathrm{QP}$ energies of $1.00 \mathrm{eV}$ and $0.89 \mathrm{eV}$, now in excellent and reasonable agreement with the PW results. All in all, we therefore conclude that the Gaussian basis set results for unoccupied states need to be considered with some caution, and Dunning correlation consistent basis sets are seemingly better suited to predict accurate values.

If we restrict the comparison between PWs and GTOs to states below the vacuum level, we find the agreement to be generally, as for the HOMO, rather satisfactory. Differences are about a factor two larger than for the HUMO, but considering the previous discussion on the possible issues with the Gaussian basis sets for unoccupied orbitals, this is certainly not astonishing.

Finally, concerning the agreement with the experiment, we find a similar absolute deviation as for the first ionization energies (compare with Tab. I). To make the comparison between the LUMO energies and the experiment more immediate, the second last column reports the negative of the experimental electron affinities, which is overall in quite satisfactory agreement with experiment.

\section{DISCUSSIONS AND CONCLUSIONS}

The main purpose of the present work is a careful comparison of $G W$ QP energies obtained using Gaussian type orbitals and plane waves. One important motivation was that the values reported for the Berkeley $G W$ code were typically $200 \mathrm{meV}$ smaller than the basis set extrapolated GTO results. However, the Gaussian basis set extrapolation also often increased the predicted QP energies by some $100 \mathrm{meV}$. Since basis set extrapolation using GTOs is not necessarily accurate, and since the Berkeley $G W$ calculations are often closer to the uncorrected values than the basis set extrapolated values, we felt that it is important to bring in a third independent set of calculations, hopefully confirming one or the other of the previous values.

The main outcome of our work is that our VASP predicted HOMOs are in excellent agreement with the basis set extrapolated GTO results. We believe this establishes beyond doubt that the values reported in Ref. 27 are very reliable and can be used as a rigorous benchmark for future implementations. In the few cases (iodine compounds, $\mathrm{Br}_{2}$ and $\mathrm{Ag}_{2}$ ), where the GTO calculations were not extrapolated to the basis set limit, we find - not unexpectedly - that the non basis set extrapolated GTO values underestimate the IP by about $300-400 \mathrm{meV}$. The present work also reports basis set extrapolated GTO values for these molecules finding good agreement with VASP PW results.

Although the mean absolute deviation between our PAW PW results and the GTO results is only $60 \mathrm{meV}$, we found larger discrepancies for molecules containing copper, fluorine and nitrogen. We traced these differences back to the use of non-normconserving PAW potentials: using normconserving PAW potentials the agreement between PW calculations and GTOs improves further. 
For the LUMO, results are slightly less satisfactory. Agreement between GTOs and PWs is good for QP energies below the vacuum level, although even for those there are more out-liners and the average deviation is larger. For instance, differences are sizable for some seemingly simple dimers. We attribute this to very large basis set corrections for GTOs for some molecules (e.g. $700 \mathrm{meV}$ for $\mathrm{As}_{2}$ ).

If the predicted QP LUMOs are above the vacuum level, the differences between the PW and GTO results can be very large and can reach $11 \mathrm{eV}(\mathrm{Ne})$. Our explanation for this behavior is that the GTO basis sets employed in Ref. 27 are not always sufficiently flexible to model unoccupied states. This is particularly true for atoms and small dimers, where the LUMO has a character that is very different from a linear combination of atomic like orbitals. In most cases, these basis set issues lead to small but noticeable errors on the level of DFT, but they are dramatically amplified at the level of $G_{0} W_{0}$. For Xe, $\mathrm{H}_{2} \mathrm{O}$ and $\mathrm{CH}_{4}$, GTO calculations with improved basis sets have been reported finding very good to good agreement with the PW results.

If we disregard the slightly disconcerting propagation of errors in going from DFT to $G_{0} W_{0}$ for LUMOs, we are satisfied by the agreement between plane waves and Gaussian type orbitals. As already stated, for the HOMO the mean absolute deviation is only $60 \mathrm{meV}$, which is excellent if one considers that the computational details are so different. Furthermore, our results have been obtained using the $G W$ PAW potentials distributed with vasp.5.4, so that similar calculations e.g. for molecules adsorbed on surfaces can be readily performed using the projector augmented wave method.

\section{ACKNOWLEDGMENTS}

This work was supported by the Austrian Science Fund (FWF) within the Spezialforschungsbereich Vienna Computational Materials Laboratory (SFB ViCoM, F41) and the Deutsche Forschungsgruppe Research Unit FOR 1346. P. Liu is grateful to the China Scholarship Council (CSC)-FWF Scholarship Program. Computational resources were provided by the Vienna Scientific Cluster (VSC) and supercomputing facilities of the Université catholique de Louvain (CISM/UCL).
[1] L. Hedin, Phys. Rev. 139, A796 (1965).

[2] W. Hanke and L. J. Sham, Phys. Rev. Lett. 43, 387 (1979)

[3] G. Strinati, H. J. Mattausch, and W. Hanke, Phys. Rev. Lett. 45, 290 (1980).

[4] G. Strinati, H. J. Mattausch, and W. Hanke, Phys. Rev. B 25, 2867 (1982).

[5] M. S. Hybertsen and S. G. Louie, Phys. Rev. B 34, 5390 (1986).

[6] M. van Schilfgaarde, T. Kotani, and S. Faleev, Phys. Rev. Lett. 96, 226402 (2006).

[7] M.Shishkin and G.Kresse, Phys. Rev. B 75, 235102 (2007).

[8] F. Fuchs, J. Furthmüller, F. Bechstedt, M. Shishkin, and G. Kresse, Phys. Rev. B 76, 115109 (2007).

[9] S. V. Faleev, M. van Schilfgaarde, and T. Kotani, Phys. Rev. Lett. 93, 126406 (2004).

[10] F. Caruso, P. Rinke, X. Ren, M. Scheffler, and A. Rubio, Phys. Rev. B 86, 081102 (2012), arXiv:1202.3547.

[11] F. Caruso, P. Rinke, X. Ren, A. Rubio, and M. Scheffler, Phys. Rev. B 88, 075105 (2013)

[12] F. Kaplan, F. Weigend, F. Evers, and M. J. van Setten, J. Chem. Theory Comput. 11, 5152 (2015).

[13] F. Kaplan, M. E. Harding, C. Seiler, F. Weigend, F. Evers, and M. J. van Setten, J. Chem. Theory Comput. 12, 2528 (2016).

[14] J. Lischner, S. Sharifzadeh, J. R. Deslippe, J. B. Neaton, and S. G. Louie, Phys. Rev. B 90, 115130 (2014), arXiv:arXiv:1409.2901v1,

[15] P. Koval, D. Foerster, and D. Sánchez-Portal, Phys. Rev. B 89, 155417 (2014), arXiv:1404.1715.

[16] X. Ren, P. Rinke, V. Blum, J. Wieferink, A. Tkatchenko, A. Sanfilippo, K. Reuter, and M. Scheffler, New J. Phys. 14, 053020 (2012).
[17] X. Blase, C. Attaccalite, and V. Olevano, Phys. Rev. B 83, 115103 (2011)

[18] F. Bruneval, J. Chem. Phys. 136, 194107 (2012)

[19] M. J. van Setten, F. Weigend, and F. Evers, J. Chem. Theory Comput. 9, 232 (2013).

[20] J. Wilhelm, M. Del Ben, and J. Hutter, J. Chem. Theory Comput. 12, 3623 (2016).

[21] D. Foerster, P. Koval, and D. Sánchez-Portal, J. Chem. Phys. 135, 074105 (2011)

[22] S. H. Ke, Phys. Rev. B - Condens. Matter Mater. Phys. 84, 205415 (2011), arXiv:1012.1084.

[23] J. Laflamme Janssen, B. Rousseau, and M. Cote, Phys. Rev. B 91, 125120 (2015).

[24] M. Govoni and G. Galli, J. Chem. Theory Comput. 11, 2680 (2015), http://dx.doi.org/10.1021/ct500958p.

[25] J. Klimeš, M. Kaltak, and G. Kresse, Phys. Rev. B 90, 075125 (2014), arXiv:arXiv:1404.3101v1.

[26] F. Bruneval and M. A. L. Marques, J. Chem. Theory Comput. 9, 324 (2013).

[27] M. J. van Setten, F. Caruso, S. Sharifzadeh, X. Ren, M. Scheffler, F. Liu, J. Lischner, L. Lin, J. R. Deslippe, S. G. Louie, C. Yang, F. Weigend, J. B. Neaton, F. Evers, and P. Rinke, J. Chem. Theory Comput. 11, 5665 (2015), pMID: 26642984.

[28] J. Deslippe, G. Samsonidze, D. a. Strubbe, M. Jain, M. L. Cohen, and S. G. Louie, Comput. Phys. Commun. 183, 1269 (2012), arXiv:1111.4429

[29] A. Schindlmayr, Phys. Rev. B 87, 075104 (2013) arXiv:arXiv:1302.6368v1

[30] B.-C. Shih, Y. Xue, P. Zhang, M. L. Cohen, and S. G. Louie, Phys. Rev. Lett. 105, 146401 (2010).

[31] C. Friedrich, M. C. Müller, and S. Blügel, Phys. Rev. B 83, 081101 (2011), ibid 84, 039906(E) (2011).

[32] F. Bruneval and X. Gonze, Phys. Rev. B - Condens. 
Matter Mater. Phys. 78, 1 (2008)

[33] F. Bruneval, N. Vast, and L. Reining, Phys. Rev. B 74, $045102(2006)$

[34] M. Shishkin and G. Kresse, Phys. Rev. B 74, 035101 (2006).

[35] M. Kaltak, J. Klimeš, and G. Kresse, J. Chem. Theory Comput. 10, 2498 (2014)

[36] M. Kaltak, J. Klimeš, and G. Kresse, Phys. Rev. B 90, 054115 (2014)

[37] P. Liu, M. Kaltak, J. Klimeš, and G. Kresse, Phys. Rev. B 94, 165109 (2016), arXiv:1607.02859.

[38] G. A. J. Baker, Essentials of Padé Approximants (Academic Press, New York, 1975) Chap. 18.

[39] J. Harl and G. Kresse, Phys. Rev. B 77, 045136 (2008).

[40] J. J. Shepherd, A. Grüneis, G. H. Booth, G. Kresse, and A. Alavi, Phys. Rev. B 86, 035111 (2012).

[41] T. Björkman, A. Gulans, A. V. Krasheninnikov, and R. M. Nieminen, Phys. Rev. Lett. 108, 235502 (2012).

[42] C. A. Rozzi, D. Varsano, A. Marini, E. K. U. Gross, and A. Rubio, Phys. Rev. B 73, 205119 (2006)

[43] A. Baldereschi and E. Tosatti, Phys. Rev. B 17, 4710 (1978).

[44] M. Gajdoš, K. Hummer, G. Kresse, J. Furthmüller, and F. Bechstedt, Phys. Rev. B 73, 045112 (2006).

[45] G. Makov and M. Payne, Phys. Rev. B 51, 4014 (1995).

[46] R. L. Kelly, J. Phys. Chem. Ref. Data 16, Supplement 1 (1987).

[47] K.-M. Weitzel, J. Mähnert, and M. Penno, Chem. Phys. Lett. 224, 371 (1994)

[48] R. C. Wetzel, F. A. Baiocchi, T. R. Hayes, and R. S. Freund, Phys. Rev. A 35, 559 (1987).

[49] H. Schäfer and H. Rabeneck, Zeitschrift für Anorg. und Allg. Chemie 545, 224 (1987)

[50] E. McCormack, J. M. Gilligan, C. Cornaggia, and E. E. Eyler, Phys. Rev. A 39, 2260 (1989)

[51] P. Dugourd, D. Rayane, P. Labastie, B. Vezin, J. Chevaleyre, and M. Broyer, Chem. Phys. Lett. 197, 433 (1992)

[52] M. M. Kappes, P. Radi, M. Schär, and E. Schumacher, Chem. Phys. Lett. 113, 243 (1985).

[53] A. Herrmann, S. Leutwyler, E. Schumacher, and L. Wöste, Helv. Chim. Acta 61, 453 (1978).

[54] T. Trickl, E. F. Cromwell, Y. T. Lee, and A. H. Kung, J. Chem. Phys. 91 (1989).

[55] D. K. Bulgin, J. M. Dyke, and A. Morris, J. Chem. Soc. Faraday Trans. 2 72, 2225 (1976).

[56] K. H. Lau, R. D. Brittain, and D. L. Hildenbrand, J. Phys. Chem. 86, 4429 (1982).

[57 H. V. Lonkhuyzen and C. A. D. Lange, Chem. Phys. 89, $313(1984)$.

[58] J. M. Dyke, G. D. Josland, J. G. Snijders, and P. M. Boerrigter, Chem. Phys. 91, 419 (1984)

[59] K. Kimura, S. Katsumata, Y. Achiba, T. Yamazaki, and S. Iwata, in Handb. HeI Photoelectron Spectra Fundam. Org. Compd. (Japan Scientific Soc. Press, Tokyo, 1981).

[60] G. Bieri and L. Åsbrink, J. Electron Spectros. Relat. Phenomena 20, 149 (1980)

[61] R. Ramanathan, J. A. Zimmerman, and J. R. Eyler, J. Chem. Phys. 98, 7838 (1993).

[62] V. Plemenkov, Y. Villem, N. Villem, I. Bolesov, L. Surmina, N. Yakushkina, and A. Formanovskii, Zh. Obs. Khim. 51, 2076 (1981).

[63] J. O. Howell, J. M. Goncalves, C. Amatore, L. Klasinc,
R. M. Wightman, and J. K. Kochi, J. Am. Chem. Soc. 106, 3968 (1984)

[64] E. W. Fu and R. C. Dunbar, J. Am. Chem. Soc. 100, $2283(1978)$

[65] V. Kiselev, A. Sakhabutdinov, I. Shakirov, V. Zverev, and A. Konovalov, Zh. Org. Khim. 28, 2244 (1992).

[66] G. Bieri, W. von Niessen, L. Åsbrink, and A. Svensson, Chem. Phys. 60, 61 (1981)

[67] R. Cambi, G. Ciullo, A. Sgamellotti, F. Tarantelli, R. Fantoni, A. Giardini-guidoni, I. E. McCarthy, and V. di Martino, Chem. Phys. Lett. 101, 477 (1983)

[68] K. Wittel, H. Bock, and R. Manne, Tetrahedron 30, 651 (1974).

[69] G. Bieri, L. Åsbrink, and W. V. Niessen, J. Electron Spectros. Relat. Phenomena 23, 281 (1981).

[70] R. N. Dixon, J. N. Murrell, and B. Narayan, Mol. Phys. 20, 611 (1971)

[71] G. Jonkers, C. Lange, and J. G. Snijders, Chem. Phys. 69, 109 (1982)

[72] R. Roberge, C. Sandorfy, J. I. Matthews, and O. P. Strausz, J. Chem. Phys. 69, 5105 (1978).

[73] A. W. Potts and W. C. Price, Proc. R. Soc. London A Math. Phys. Eng. Sci. 326, 165 (1972).

[74] H. Bock, W. Ensslin, F. Feher, and R. Freund, J. Am. Chem. Soc. 98, 668 (1976).

[75] "NIST Chemistry WebBook," (2015).

[76] M. Farber, R. D. Srivastava, and J. W. Moyer, J. Chem. Thermodyn. 14, 1103 (1982)

[77] B. Ruscic, C. A. Mayhew, and J. Berkowitz, J. Chem. Phys. 88, 5580 (1988).

[78] L. Asbrink, A. Svensson, W. von Niessen, and G. Bieri, J. Electron Spectros. Relat. Phenomena 24, 293 (1981)

[79] H. Baumgaertel, H. W. Jochims, E. Ruehl, H. Bock, R. Dammel, J. Minkwitz, and R. Nass, Inorg. Chem. 28, 943 (1989).

[80] T. Cvitaš and L. Klasinc, J. Chem. Soc. Faraday Trans. 2 72, $1240(1976)$

[81] A. H. Cowley, R. A. Kemp, M. Lattman, and M. L. McKee, Inorg. Chem. 21, 85 (1982)

[82] R. Demuth, Zeitschrift für Naturforsch. B 32, 1252 (1977)

[83] G. Bieri, L. Åsbrink, and W. von Niessen, J. Electron Spectros. Relat. Phenomena 27, 129 (1982).

84] M. S. Banna and D. A. Shirley, J. Chem. Phys. 63, 4759 (1975).

[85] R. Wang, M. A. Dillon, and D. Spence, J. Chem. Phys. 80, 63 (1984)

[86] J. Berkowitz, H. A. Tasman, and W. A. Chupka, J. Chem. Phys. 36, 2170 (1962)

[87] D. L. Hildenbrand, J. Chem. Phys. 48, 3657 (1968).

[88] J. M. Dyke, D. Haggerston, A. Wright, A. Morris, E. van Lenthe, and J. Snijders, J. Electron Spectros. Relat. Phenomena 85, 23 (1997)

[89] J. M. Dyke, C. Kirby, A. Morris, B. Gravenor, R. Klein, and P. Rosmus, Chem. Phys. 88, 289 (1984).

[90] M. Farber and R. D. Srivastava, J. Chem. Phys. 81, 241 (1984).

[91] E. R. Fisher, B. L. Kickel, and P. Armentrout, J. Chem. Phys. 97, 4859 (1992)

[92] A. W. Potts and W. C. Price, Phys. Scr. 16, 191 (1977).

[93] O. Grabandt, R. Mooyman, and C. De Lange, Chem. Phys. 143, 227 (1990)

[94] E. P. F. Lee and A. W. Potts, Proc. R. Soc. A Math. Phys. Eng. Sci. 365, 395 (1979) 
[95] G. K. Barker, M. F. Lappert, J. B. Pedley, G. J. Sharp, and N. P. C. Westwood, J. Chem. Soc. Dalt. Trans. , 1765 (1975)

[96] J. Kreile, A. Schweig, and W. Theil, Chem. Phys. Lett. 87, 473 (1982)

[97] D. K. Bulgin, J. M. Dyke, and A. Morris, J. Chem. Soc., Faraday Trans. 2 73, 983 (1977).

[98] V. Vovna, F. Vilesov, and S. Lopatin, Opt. Spectrosc. 38, 143 (1975).

[99] K. Ohno, K. Okamura, H. Yamakado, S. Hoshino, T. Takami, and M. Yamauchi, J. Phys. Chem. 99, 14247 (1995)

[100] A. S. Vorob'ev, I. I. Furlei, A. S. Sultanov, V. I. Khvostenko, G. V. Leplyanin, A. R. Derzhinskii, and G. A. Tolstikov, Bull. Acad. Sci. USSR Div. Chem. Sci. 38, 1388 (1989).

[101] K. Ohno, K. Imai, and Y. Harada, J. Am. Chem. Soc. 107, 8078 (1985)

[102] K. Johnson, I. Powis, and C. Danby, Chem. Phys. 70, 329 (1982)

[103] W. von Niessen, G. Bieri, and L. Åsbrink, J. Electron Spectros. Relat. Phenomena 21, 175 (1980)

[104] F. S. Ashmore and A. R. Burgess, J. Chem. Soc. Faraday Trans. 2 73, 1247 (1977)

[105] J. H. D. Eland and J. Berkowitz, J. Chem. Phys. 67, 5034 (1977)

[106] A. Schweig and W. Thiel, Mol. Phys. 27, 265 (1974).

[107] A. Potts and T. Williams, J. Electron Spectros. Relat. Phenomena 3, 3 (1974)

[108] S. Cradock and W. Duncan, J. Chem. Soc. Faraday Trans. 2 71, 1262 (1975)

[109] S. Katsumata, H. Shiromaru, and T. Kimura, Bull. Chem. Soc. Jpn. 57, 1784 (1984)

[110] L. P. Theard and D. L. Hildenbrand, J. Chem. Phys. 41, 3416 (1964).

[111] N. Dalleska and P. Armentrout, Int. J. Mass Spectrom. Ion Process. 134, 203 (1994)

[112] R. Ballard, J. Jones, D. Read, A. Inchley, and M. Cranmer, Chem. Phys. Lett. 137, 125 (1987).

[113] G. Furin, A. Sultanov, and I. I. Furlei, Dokl. Phys. Chem. 3, 530 (1987).

[114] T. Kobayashi and S. Nagakura, J. Electron Spectros. Relat. Phenomena 4, 207 (1974).

[115] N. Hush and A. S. Cheung, Chem. Phys. Lett. 34, 11 (1975)

[116] J. Lin, C. Yu, S. Peng, I. Akiyama, K. Li, L. K. Lee, and P. R. LeBreton, J. Am. Chem. Soc. 102, 4627 (1980)

[117] D. Dougherty, K. Wittel, J. Meeks, and S. P. McGlynn, J. Am. Chem. Soc. 98, 3815 (1976).

[118] M. H. Palmer, I. Simpson, and R. J. Platenkamp, J. Mol. Struct. 66, 243 (1980)

[119] V. Beutel, H.-G. Kramer, G. L. Bhale, M. Kuhn, K. Weyers, and W. Demtroder, J. Chem. Phys. 98, 2699 (1993)

[120] K. Franzreb, A. Wucher, and H. Oechsner, Zeitschrift fr Phys. D Atoms, Mol. Clust. 17, 51 (1990)
[121] T. Kato, Comm. Pure Appl. Math. 10, 151 (1957)

[122] P. Scherpelz, M. Govoni, I. Hamada, and G. Galli, J. Chem. Theory Comput. 12, 3523 (2016).

[123] R. C. Weast and M. J. Astle, CRC Handbook of Chemistry and Physics, 92nd ed. (CRC Press, New York, 2011).

[124] K. M. McHugh, J. G. Eaton, G. H. Lee, H. W. Sarkas, L. H. Kidder, J. T. Snodgrass, M. R. Manaa, and K. H. Bowen, J. Chem. Phys. 91 (1989).

[125] R. O. Jones, G. Ganteför, S. Hunsicker, and P. Pieperhoff, J. Chem. Phys. 103, 9549 (1995).

[126] T. P. Lippa, S.-J. Xu, S. A. Lyapustina, J. M. Nilles, and K. H. Bowen, J. Chem. Phys. 109 (1998).

[127] J. A. Ayala, W. E. Wentworth, and E. C. M. Chen, J. Phys. Chem. 85, 768 (1981)

[128] D. W. Arnold, S. E. Bradforth, T. N. Kitsopoulos, and D. M. Neumark, J. Chem. Phys. 95, 8753 (1991).

[129] T. M. Miller, A. A. Viggiano, and A. E. S. Miller, J. Phys. Chem. A 106, 10200 (2002)

[130] H. W. Sarkas, J. H. Hendricks, S. T. Arnold, and K. H. Bowen, J. Chem. Phys. 100, 1884 (1994)

[131] C. T. Wickham-Jones, S. Moran, and G. B. Ellison, J. Chem. Phys. 90, 795 (1989)

[132] O. Boltalina, A. Borshchevskii, L. Sidorov, and V. Chepurnykh, Zh. Fiz. Khim. SSSR 65, 928 (1991).

[133] A. E. S. Miller, T. M. Miller, A. A. Viggiano, R. A. Morris, J. M. Van Doren, S. T. Arnold, and J. F. Paulson, J. Chem. Phys. 102, 8865 (1995)

[134] T. M. Miller, D. G. Leopold, K. K. Murray, and W. C. Lineberger, J. Chem. Phys. 85, 2368 (1986).

[135] S. J. Cavanagh, S. T. Gibson, and B. R. Lewis, J. Chem. Phys. 137, 144304 (2012)

[136] D. W. Arnold, C. Xu, E. H. Kim, and D. M. Neumark, J. Chem. Phys. 101, 912 (1994)

[137] M. R. Nimlos and G. B. Ellison, J. Phys. Chem. 90, 2574 (1986)

[138] S. N. Eustis, D. Wang, K. H. Bowen, and G. Naresh Patwari, J. Chem. Phys. 127, 114312 (2007)

[139] J. Schiedt, R. Weinkauf, D. M. Neumark, and E. Schlag, Chem. Phys. 239, 511 (1998).

[140] K. Aflatooni, G. A. Gallup, and P. D. Burrow, J. Phys. Chem. A 102, 6205 (1998).

[141] H. Handschuh, C. Cha, P. S. Bechthold, G. Gantefor, and W. Eberhardt, J. Chem. Phys. 102, 6406 (1995).

[142] K. J. Taylor, C. L. Pettiette-Hall, O. Cheshnovsky, and R. E. Smalley, J. Chem. Phys. 96, 3319 (1992).

[143] X. Wu, Z. Qin, H. Xie, R. Cong, X. Wu, Z. Tang, and H. Fan, J. Phys. Chem. A 114, 12839 (2010).

[144] L. a. Curtiss, P. C. Redfern, and K. Raghavachari, J. Chem. Phys. 126, 084108 (2007).

[145] J. T. Golab, B. S. Thies, D. L. Yeager, and J. a. Nichols, J. Chem. Phys. 84, 284 (1986)

[146] S. Rayne and K. Forest, Comput. Theor. Chem. 974, $163(2011)$. 